\title{
CONTROL DE MANIPULADORES TELEOPERADOS
}

\section{TELEOPERATED MAYIPULATOR COMTROL}

Fecha de Recepción: 16 de mayo de 2006

Fecha de Aprobación: 27 de junio de 2006

\author{
Ricardo Enrique Gutiérrez Carvajal ${ }^{1}$ \\ Ricardo Andrés Castillo Estepa ${ }^{2}$
}

\section{RESUMEN}

Este trabajo presenta una revisión bibliográfica acerca de distintos modelos utilizados en el diseño de controladores para manipuladores teleoperados, se realiza una descripción tanto de las ventajas como de los problemas que se presentan al momento de implementar sistemas de control con técnicas convencionales e inteligentes (Redes Neuronales y Lógica Difusa) en estos dispositivos. También se abordan descriptivamente los sistemas teleoperados, para finalmente presentar una sección especializada en la que se muestran estrategias de control empleadas para tratar los retardos de señal inherentes a la teleoperación de manipuladores. En la última sección se citan algunas aplicaciones importantes para estos sistemas.

Palabras clave: Teleoperación, Control, Lógica Difusa, Redes Neuronales, Manipuladores antropomórficos.

\section{ABSTRACT}

This work is a bibliographical review about different models used to design teleoperated manipulator controllers, there is a quick description of some advantages and disadvantages to implement conventional or intelligent techniques (Neural Networks and Furay Logic) to develop control systems applied to these devices. In addition, here the teleoperation systems are descriptively presented; finally there is a specialized section that shows some control strategies in order to deal with signal delays, inherent to manipulator teleoperation. In the last section some important applications for these systems are described.

Key Words: Teleoperation, Control, Fuг:y Logic, Neural Networks, Antbropomorphic Manipulators. 


\section{INTRODUCCIÓN}

Este artículo pretende realizar una revisión bibliográfica acerca del control de manipuladores antropomórficos teleoperados, para esto se divide el tema de este documento en dos grandes partes: control de manipuladores y teleoperación de manipuladores.

A lo largo de la historia el ser humano ha buscado desarrollar herramientas para manipular su entorno. La teleoperación tiene sus orígenes en la industria nuclear, allí un operario, desde un lugar seguro, podía llevar a cabo operaciones de manipulación de sustancias peligrosas sin riesgo para él. Pero fue a partir de 1947 cuando surgieron trabajos para implementar un esquema completo de telemanipulación, se desarrollaron entonces dispositivos sincronizados mecánicamente denominados maestro-esclavo, a los cuales se les acoplo realimentación de fuerza en 1954. En los años 60 al utilizarse esta tecnología por primera vez en aplicaciones submarinas y espaciales, se revela la presencia de grandes retrasos en el control, lo cual acarrea inestabilidad en el sistema teleoperado.

Un sistema teleoperado se compone de: un dispositivo maestro que realiza la lectura de los movimientos de un operario, un dispositivo esclavo o robot el cual realiza tareas en un ambiente remoto, canales de comunicación, interfaz con el usuario y sistemas de realimentación de datos, de forma visual o por realimentación de posición del dispositivo esclavo. La Figura 1 ilustra la topología del problema de la telemanipulación En la sección II se realiza una inspección general al problema de la teleoperación de manipuladores, en la sección III se realiza un muestran ge- neralidades del control de manipuladores no teleoperados, en la sección IV se hace una revisión de trabajos sobre control convencional aplicado a manipuladores, en la sección $V$ se introduce el concepto de controladores basados en técnicas de inteligencia artificial, enfatizando en aquellos que utilizan lógica difusa y redes neuronales. La sección VI discute la aplicación de algunas de estas técnicas a la teleoperación de sistemas. Luego, en la sección VII se muestran algunos avances en el control de telemanipuladores y se plantea la problemática de los retardos de señal. En la sección VIII se describen algunas aplicaciones de robots teleoperados y finálmente en la sección IX se formulan algunas conclusiones acerca de esta revisión bibliográfica.

\section{DESCRIPCIÓYY DEL PROBLEMA}

Los manipuladores son utilizados para diversas aplicaciones, entre las que se cuentan: la industria automotriz, como soldadoras y ensambladoras, la industria espacial, lugares donde la presencia humana se hace riesgosa, como manipulación de material nuclear y/o químico, desarrollo de tareas en sitios de difícil alcance, etc. Una de las configuraciones del dispositivo esclavo mas utilizada, es la de manipulador antropomórfico de mas de tres grados de libertad; esta configuración presenta un comportamiento altamente no lineal, debido a que la inercia vista por cada actuador esta cambiando en razón de la posición del efector final, además debido a que muchos de estos manipuladores deben ser teleoperados remotamente para realizar tareas no preestablecidas, un operario a cargo del control manual del robot debe contar con la mayor cantidad de datos de realimentación, que indiquen como se esta desarrollando el trabajo realizado por el manipulador 
en cualquier momento. En este tipo de sistemas se presentan retardos de señal no constantes y difíciles de manejar por técnicas convencionales como es el caso de la información que viaja a través de Internet.

Cuando se realiza teleoperación, la forma mas fiel en la que un operario puede percibir lo que ocurre en el ambiente remoto, es experimentando el mismo, las reacciones en el manipulador, para esto se realiza un sensado de las fuerzas soportadas por las articulaciones del robot, para luego realimentarlas al dispositivo maestro. No obstante el retraso de la señal de control en estos sistemas, puede acarrear un comportamiento inestable en el dispositivo esclavo.

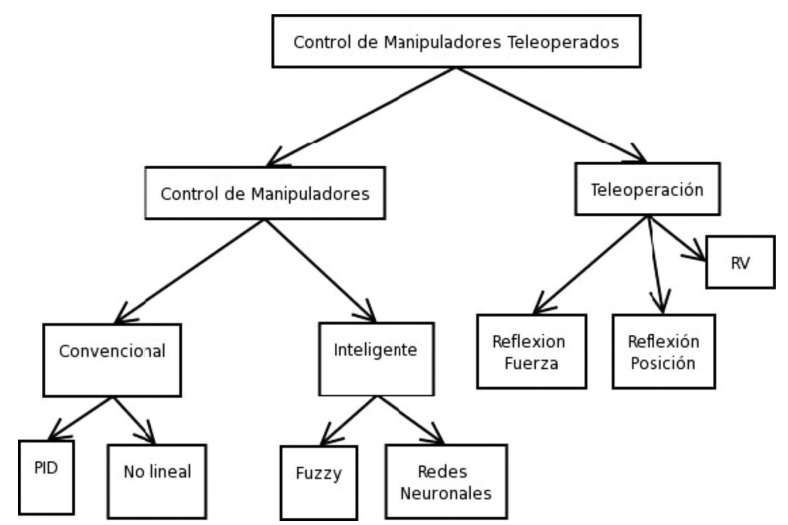

Figura 1. Topología del problema de teleoperación de manipuladores
Para solucionar estos inconvenientes se trabaja en el diseño de un controlador suficientemente robusto, que maneje: la retroalimentación de fuerza del dispositivo remoto a uno local, la no linealidad de la planta y retraso de la señal entre dispositivo maestro y esclavo. La Figura 2 muestra el diagrama en bloques de un sistema de control con retrasos.

\section{CONTROL DE MANIPULADORES}

A los robots antropomórficos se les demanda elevadas prestaciones de velocidad y precisión de movimiento. Por esto encontrar el control más eficiente para este tipo de manipuladores es una de las tareas más importantes en la robótica moderna. El control dinámico debe procurar que las trayectorias planeadas sean seguidas fielmente por el robot, para esto es necesario tener conocimiento del modelo dinámico del robot y de las herramientas de diseño aportadas por la teoría de control [1]. El diagrama de control típico se puede apreciar en la Figura 3.

El modelo dinámico de los manipuladores antropomórficos es fuertemente no lineal, multivariable, acoplado y de parámetros variables, lo que hace que el diseño del control para este tipo de mecanismos sea muy complejo. En la práctica se realizan simplificaciones limitando ciertas

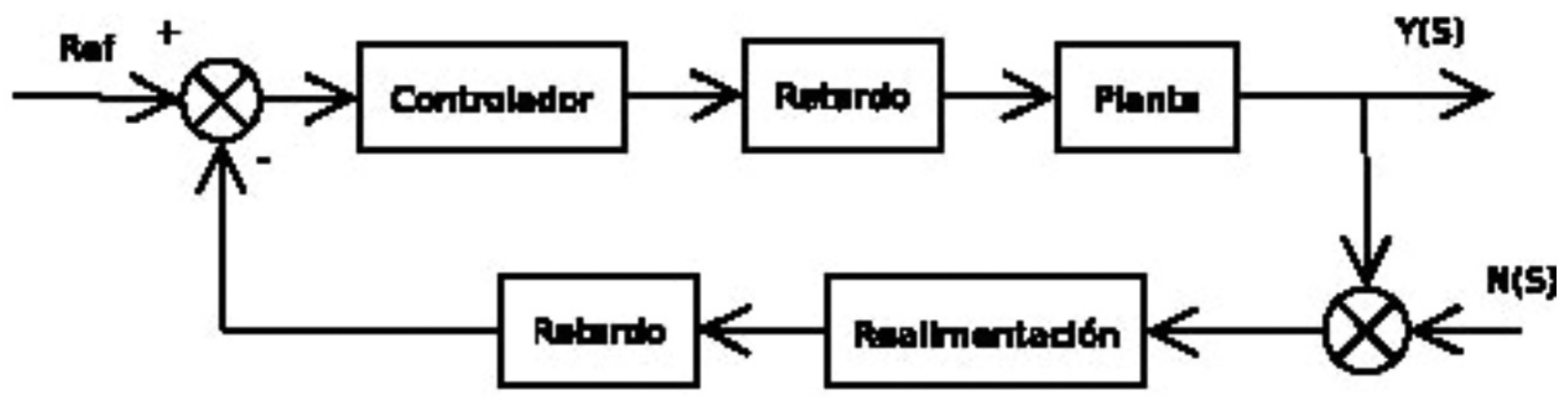

Figura 2. Diagrama en bloques del sistema de control con retardos de señal 


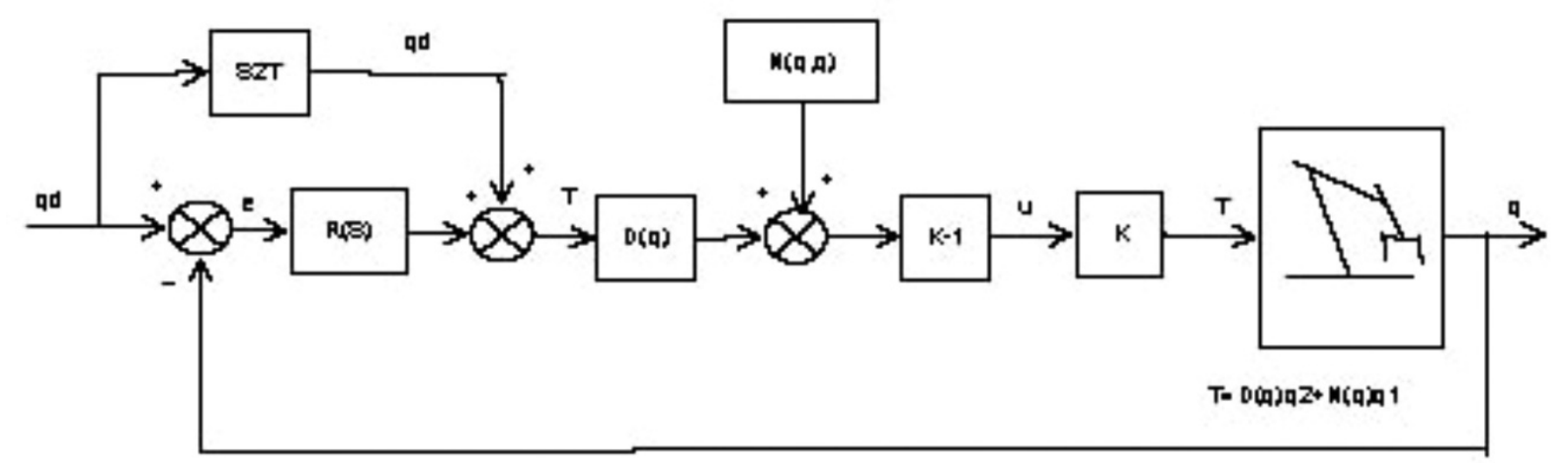

Figura 3. Diagrama del sistema de control de un manipulador [2]

características de movimiento del robot, y aunque el uso de técnicas más robustas puede mejorar este problema, su implementación aumenta considerablemente el costo computacional. En la actualidad existen técnicas de control convencional, entendiendo por convencional aquellas que se basan en ecuaciones diferenciales, tanto lineales como no lineales, que pueden ser utilizadas en el control de robots antropomórficos, en este caso, el manipulador debe restringir su funcionamiento a rangos pequeños de movimiento, donde se pueda asumir un comportamiento lineal del mismo; en caso de no restringir el rango de movimiento del robot, se necesitan métodos mas pesados computacionalmente. Se puede afirmar que ambos casos se comportan bien ante modelos dinámicos bien conocidos; pero cuando aparecen parámetros desconocidos, generalmente por la simplificación del modelo, como variables imprecisas o difíciles de medir: rozamiento entre articulaciones, inercia de las mismas; o porque el cálculo del modelo inverso es excesivamente lento [2], es necesario utilizar controles adaptativos.

Entre las técnicas de control adaptativo no inteligentes se encuentran: El Control Adaptativo por planificación de ganancias, en el cual se divi- de el funcionamiento del robot en varios rangos de operación, definidos cada uno por la posición y carga del manipulador, y luego se implementa un sistema de control convencional para cada rango; este tipo de controlador presenta como inconveniente principal la existencia de muchísimos puntos de funcionamiento, lo que complica excesivamente el diseño del mismo. En el Control adaptativo con modelo de referencia (MRAC), el controlador utiliza un modelo de referencia, generalmente difícil de elegir, que tiene un comportamiento ideal; en este caso, el comportamiento instantáneo del modelo real y del modelo deseado son utilizados para adaptar el controlador [2].

Otra familia de controladores adaptativos, la constituyen los controladores inteligentes, cuyo desarrollo se ha incrementado en los últimos 30 años, a la par con las técnicas de inteligencia artificial utilizadas para su diseño, aquí se tiene como ventaja principal, el que no es necesario conocer completamente la planta, incluso en algunos casos esta puede ser completamente desconocida [3]. Entre las técnicas de IA mas utilizadas en estos controladores, se encuentran la Lógica Difusa, la cual requiere el conocimiento inicial de un experto y no precisa de tiempos de entrenamiento; el Perceptrón Multicapa (MLP), 
que es una técnica basada en el funcionamiento de las neuronas del cerebro, esta requiere el entrenamiento de una red neuronal artificial, antes de ser implementada en el controlador. CMAC, es otro tipo de red neuronal, basada en el funcionamiento del cerebelo [4], también requiere entrenamiento antes de ponerse en funcionamiento y al igual que el Perceptrón multicapa, no requiere conocimiento apriori de un experto. También es posible implementar un control con técnicas híbridas como: ANFIS, P-CMAC, PID difuso, $\mathrm{H}$-inifinito difuso, en las que se combina redes neuronales con lógica difusa.

\section{CONTROL CONVENCIONAL}

Se realiza control de los sistemas para asegurar que sigan fielmente una referencia dada, al incorporar un lazo de realimentación al control de dispositivos se puede mejorar la sensibilidad y la estabilidad del sistema. En el caso de manipuladores existe un gran numero de trabajos relacionados; la dificultad para el control convencional radica en la alta no linealidad de los modelos dinámicos de los manipuladores de varios grados de libertad y en la necesidad de conocer el sistema dinámico de la planta suficientemente.

En [5] se propone una nueva estrategia de control sin realimentación, para un manipulador flexible, el autor realiza simulaciones para un manipulador de un grado de libertad y muestra que para este caso específico, el sistema diseñado obtiene error de posición inferior al $5 \%$. Este tipo de control no es muy utilizado, debido a que no se obtienen las ventajas de utilizar un lazo realimentado en el diseño del controlador.

Para el control de manipuladores se prefiere el control retroalimentado de lazo cerrado, debido a que este permite mejorar la sensibilidad, tiempo de respuesta y estabilidad del sistema. Uno de las estructuras de control más utilizadas es el famoso controlador tipo PID, utilizado en los trabajos de Moschini [6] como punto de comparación con controladores inteligentes, debido a la conocida robustez de este tipo sistemas.

Los sistemas de control también son utilizados para reducir perturbaciones, dado que en robótica moderna se trabaja con estructuras muy livianas, en las cuales, el diseñador no solo se preocupa del seguimiento de una referencia de velocidad o posición, además debe cuidar que el robot sufra la menor cantidad de perturbaciones, muy comunes en este tipo de sistemas [7].

Otra variable que se acostumbra controlar en manipuladores, es la fuerza en cada junta [8], ya que cuando las fuerzas actuantes sobre cada articulación varían rápidamente, la potencia requerida por los actuadores aumenta, reduciéndo la vida útil de estos.

Cada una de las estrategias de control convencional, es una solución de la ecuación del Jacobiano [9] del manipulador, en donde se busca minimizar el error de posición del robot, el consumo de potencia de los actuadores y en el caso de estructuras livianas la vibración del sistema.

\section{CONTROL INTELIGENTE}

La aplicación de inteligencia artificial (IA) en la teoría de control lleva más de tres décadas. Los tópicos en que más progreso se ha logrado son la lógica difusa, que esta basada en conocimiento del experto y reglas semánticas, y las re-des neuronales artificiales, que son un modelo simplificado 
del comportamiento de las neuronas reales, cuya ventaja esta dada en su capacidad de aprender de la experiencia suministrada. A continuación se ilustran estos conceptos aplicados a la teoría de control en manipuladores.

\section{A. Control con Lógica Difusa}

La lógica difusa tiene como ventaja principal su simplicidad para trabajar problemas complejos, como lo son los controladores de sistemas no lineales. Por medio de reglas semánticas establecidas por un experto, se pueden compensar los parámetros desconocidos de un modelo y manejar las no linealidades, además la lógica difusa se puede combinar con la robustez de técnicas convencionales como el PI y el PID.

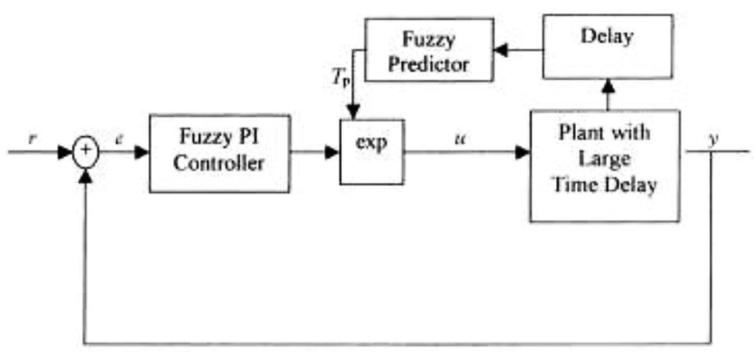

Figura 4. Diagrama del sistema de control difuso [14]

En el trabajo de Shaochenge [10] se une el poder semántico de la lógica difusa con la técnica $\mathrm{H}-\infty$ en plantas desconocidas altamente no lineales, en este caso no se requieren variables de estado; este mismo resultado se puede ver en el trabajo realizado por Tong [11]. Otra manera de compensar parámetros desconocidos es utilizando el modelo difuso de Takagi-Sugeno. [12], ampliamente usado para redes tipo ANFIS. En el trabajo de Ham [13] se diseña un controlador difuso aplicado a un manipulador y enfocado a resolver el problema de estabilidad, el controlador resultante se prueba por el criterio de Lyapunov y luego es comparado con un sistema de control no lineal convencional, mostrando que las técnicas de control difuso bien utilizadas, son tan o más poderosas que la técnicas convencionales, sobre todo al trabajar con plantas de parámetros desconocidos.

Otra aplicación importante de la lógica difusa, aparece cuando esta se combina con sistemas de control convencional como el PI, para el control de plantas con grandes retrasos [14], el diagrama de bloques de estos sistemas se muestra en la Figura 4 este esquema utiliza el poder semántico de la lógica difusa como predictor para compensar el retraso de la señal de control de la planta, también es utilizado con plantas lineales y no lineales con y sin grandes retrasos y con parámetros desconocidos [15] [16] [17] demostrando la gran versatilidad de la lógica difusa.

En los trabajos de Li [18] y Misir [19] se muestra un estudio del control por lógica difusa y el control de estructura variable. También se aprecia, como este tipo de controladores se comporta de manera no lineal si la planta lo es, y de manera lineal para plantas no lineales. Malki convierte [20] un controlador PID en uno PID difuso con las mismas características pero mejorando el tiempo de respuesta. Debido a las propiedades de la lógica difusa el modelo del sistema no siempre es requerido, por el contrario se considera que las reglas construidas con ayuda de un experto son suficientes, es decir, con este tipo de controlador es menos importante conocer la descripción exacta del sistema, que el comportamiento que se quiere de este [3].

También es posible hacer que las funciones de membresía utilizadas se adapten a la planta utilizando un algoritmo genético, buscando un con- 
trolador óptimo, como en los trabajos de Eskill [21] y Tang [22] que obtuvieron resultados satisfactorios al aplicar estas técnicas.

\section{B. Control con Redes Neuronales}

La utilización de redes neuronales para el control de sistemas, ofrece ventajas tales como el aprendizaje, esto indica que no es necesario conocer completamente el sistema dinámico de la planta, pero si el comportamiento que se quiere de ella. La relevancia de las redes neuronales en control, radica en que estas se adaptan a los cambios de la planta, obteniéndose un comportamiento satisfactorio en plantas variables y no lineales, como es el caso de los manipuladores. Yonung [23] propuso una red neuronal para controlar un manipulador, conocida como doble red neuronal, que converge exponenciálmente a soluciones óptimas. Este modelo de una arquitectura relativamente simple se simuló para un robot PA10, solucionando el problema de redundancias al incorporar restricciones físicas de posición y velocidad en las juntas.

$\mathrm{Al}$ adaptarse a los cambios de la planta, con redes neuronales no se requiere conocer el sistema dinámico de la misma [24], pero es necesario un entrenamiento adaptativo, pesado computacionalmente. En [25] se propuso un sistema de control basado en funciones de base radial para sistemas no lineales inciertos. Este sistema fue entrenado con el conocimiento fruto de la experiencia de procesos de control anteriores. Comparado con los controles adaptativos basados en redes neuronales comunes, los controles RBF son de orden bajo, por ello son computacionalmente más livianos, y en la práctica son puestos en marcha rápidamente.
Dentro del control neuronal, existen otras técnicas como los sistemas CMAC, ampliamente utilizados en el modelamiento de sistemas de control, debido a su velocidad de entrenamiento [26], y los sistemas P-CMAC [27] que surgen como contraposición a los sistemas ANFIS, el diagrama de bloques de este tipo de controlador se muestra en la Figura 5. Clark [29], analiza el efecto de un controlador neuronal sobre un sistema robótico, demostrando que es posible mejorar la sensibilidad otorgada con controles PID al utilizar técnicas de inteligencia artificial; esta combinación se logra al utilizar la salida de error del PID como la entrada a una red neuronal.

Las redes neuronales son muy versátiles y aplicables en diversos campos, así existen trabajos que proponen este tipo de solución para el problema de los retardos de señal variable [30], presentes en la comunicación de datos a través de medios físicos.

Finalmente, debido a su capacidad de adaptación, las redes neuronales son utilizadas para: diseñar controladores de manipuladores, optimizando diseño y robustez [31], y en sistemas que son difícilmente controlables por técnicas tradicionales como los robots livianos con muchos grados de libertad [4,32,33].

\section{SISTEMAS TELEOPERADOS}

Los sistemas teleoperados nacen como un requerimiento en la industria nuclear, donde se necesitaba manipular elementos en ambientes peligrosos para el ser humano. A partir de aquí se desarrolla esta tecnología, que luego es aplicada en la industria espacial, mostrando por primera 
vez el problema del retraso de señal, en ese entonces de unos tres segundos (dentro de la atmósfera terrestre); hoy en día se aprecia un retraso de 30 minutos en sondas enviadas a Marte.

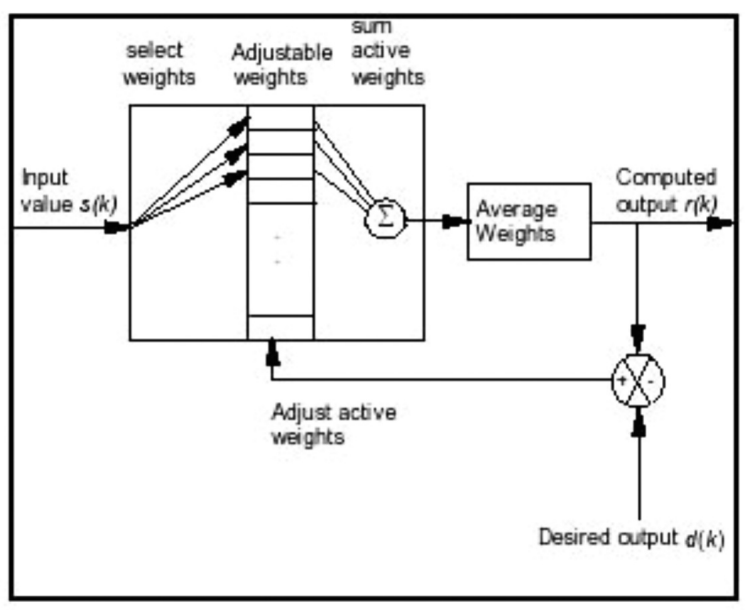

Figura 5. Diagrama en bloques del sistema de control basado en CMAC [28].

Para solucionar este problema se requieren cambios en los algoritmos y métodos de control aplicados.

Los esquemas de realimentación bilateral más utilizados son [6]

- Posición -Posición: La posición del dispositivo esclavo es determinada a partir de la posición del dispositivo maestro

- Fuerza -Posición: La posición del dispositivo esclavo se determina a partir de la posición del dispositivo maestro, pero existe una reflexión de fuerza, del dispositivo esclavo al maestro

- Fuerza -Fuerza: La posición de ambos dispositivos se determina por la lectura de los sensores de fuerza de cada uno de ellos, existe un control de posición para cada dispositivo

- Cuatro canales: Existe un intercambio de señales de posición y fuerza entre los disposi- tivos. Es el esquema que más transparencia puede dar al operario. Una visión general de algunos de estos métodos se puede apreciar en la Figura 6

\section{TELEOPERACIÓN DE MANIPULADORES}

La teleoperación es el conjunto de técnicas que permiten a un operador manejar un dispositivo remoto mediante el uso de un dispositivo local [35]. Actualmente los sistemas teleoperados tienen reflexión de fuerza, permitiendo al operario tener percepción de lo que ocurre en el ambiente remoto, este tipo de sistemas se denominan de realimentación bilateral.

Cuando los retardos de comunicación son muy grandes entre el dispositivo maestro y el esclavo, el sistema se vuelve inestable [36]. Una solución planteada es utilizar visualizadores predictivos, los cuales posibilitan al operador trabajar sobre un ambiente virtual ubicado localmente sin retrasos, que simula lo que ocurre instantes de tiempo después con el dispositivo esclavo; Cuando los retardos no son tan grandes (solo unos pocos segundos), es posible realizar un bucle de realimentación, no obstante, el dispositivo esclavo debe tener cierto nivel de autonomía.

\section{A. Control de Sistemas Teleoperados}

Cuando se hace necesaria la operación remota de un dispositivo, el algoritmo de control debe cambiarse un poco, puesto que se enfrenta a los retrasos de las señales, tanto de referencia al controlador como de realimentación de fuerza del dispositivo. Esto tiende a complicarse, debido a que en sistemas teleoperados generalmente se desea un control de seguimiento, es decir, no solo se busca la ubicación en una posición fija 
objetivo, por el contrario se necesita indicar al robot toda una trayectoria a seguir.

El control de los sistemas teleoperados maestro-esclavo generalmente incluye dos variables, posición y fuerza, las cuales son implementadas en lazos cerrados entre los dos dispositivos. Algunos métodos utilizados para esto son 0share compiant control, en el que el dispositivo esclavo posee cierta autonomía, resultando un sistema en el cual, el operador comparte la tarea de control con algunas funciones automáticas del robot esclavo, y telemonitoring sensory feedback, en el que el control de la tarea depende únicamente del operario, que además, tiene realimentación de fuerza del entorno remoto [37].

Entre los métodos convencionales usados para realizar este tipo de tarea, los más conocidos son: el método $\mathrm{H}-\infty$ [38] [11] que es independiente del retraso, y métodos predictivos [39], [14], en los cuales se anticipa la entrada de referencia dada por el operario.

Moschini [6] presentó resultados técnicos de un sistema teleoperado cuyo efector final en el dispositivo esclavo entra en contacto con una superficie, él prueba distintos algoritmos de control tales como el PID convencional, control por impedancia y un sistema híbrido entre los dos anteriores, aunque este trabajo queda en simulaciones y no se aplica nunca en un sistema real.

Otros autores trabajan con sistemas de inteligencia artificial, este el es caso de Aracil [40], quien enfoca su trabajo a la teleoperación aplicada en la industria nuclear, él propone un sistema de control basado en lógica difusa. Para mostrar los resultados de su trabajo compara este controlador con el PID convencional arrojando resultados aceptables.

Bianchi [41], se centra en el problema de los retardos variables, para esto realiza la teleoperación de un robot industrial vía Internet, mediante una página que se conecta a un programa realizado en lenguaje C.

\section{B. Sistemas de Control con Retardos de Señal}

Cuando los retrasos de señal debidos al canal de comunicación, protocolo o inclusive a la distancia son muy pequeños, se puede obviar este problema al manejarlo como un sistema de control sin retraso; los retrasos de señal son comunes cuando el dispositivo esclavo se encuentra a grandes distancias del dispositivo maestro o cuando el medio de comunicación presenta un ancho de banda muy pequeño para la transmisión de datos. Como se dijo en la sección VI, la reflexión de fuerza da mayor conocimiento al operario acerca del entorno remoto; aunque cuando los retardos son grandes, el usuario se puede confundir debido a que la información recibida de la realimentación no corresponde con la percibida por medios visuales, presentándose inestabilidad en el conjunto maestro -esclavo.

Cuando el retraso es pequeño es posible utilizar el método propuesto por Ferrell [36], donde se utiliza la técnica mover y luego esperar, en la cual el operario realiza el movimiento y luego espera a que este se vea reflejado en la pantalla antes de continuar, este es un método válido para retardos menores a 0.3 segundos.

Otro método muy común para tratar con retrasos de señal muy grandes, es utilizar reali- 


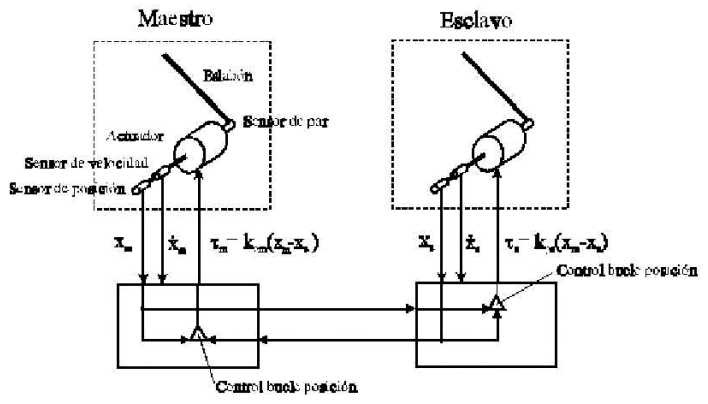

(a) Sistema posición - posición

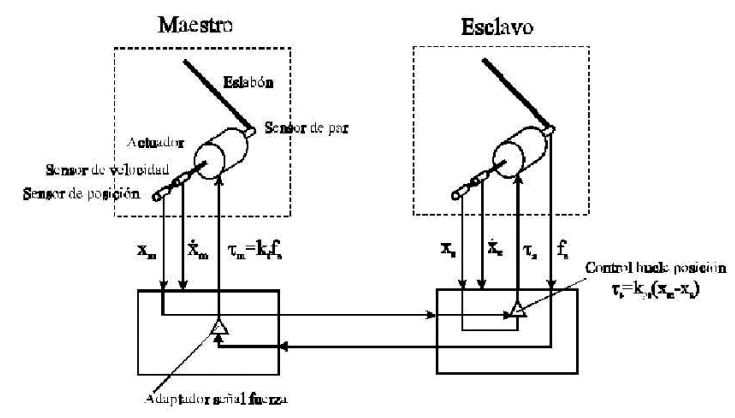

(c) Sistema fuerza - posición

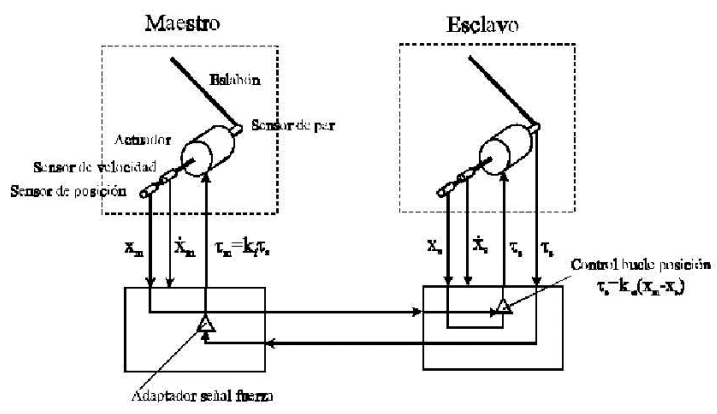

(e) Sistema fuerza - fuerza

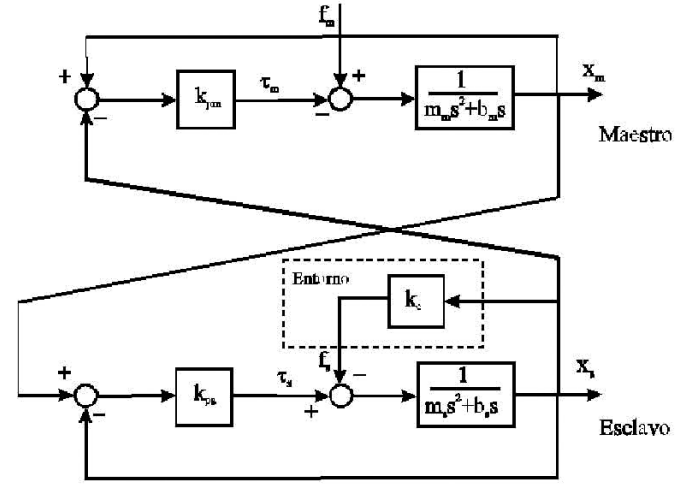

(b) Diagrama de control posición posición

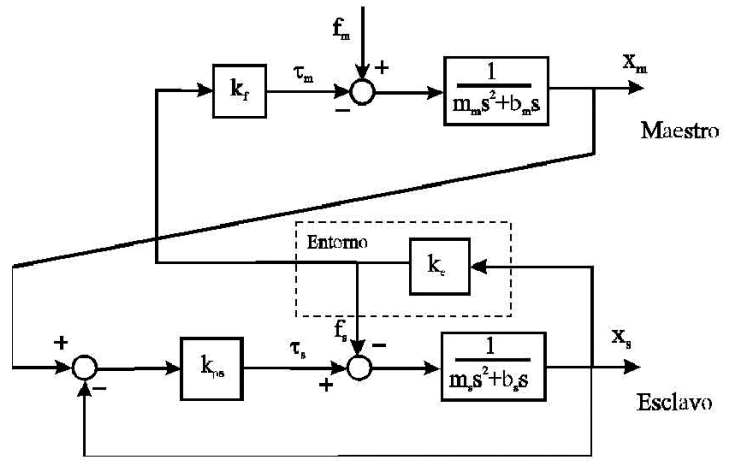

(d) Diagrama de control fuerza - posición

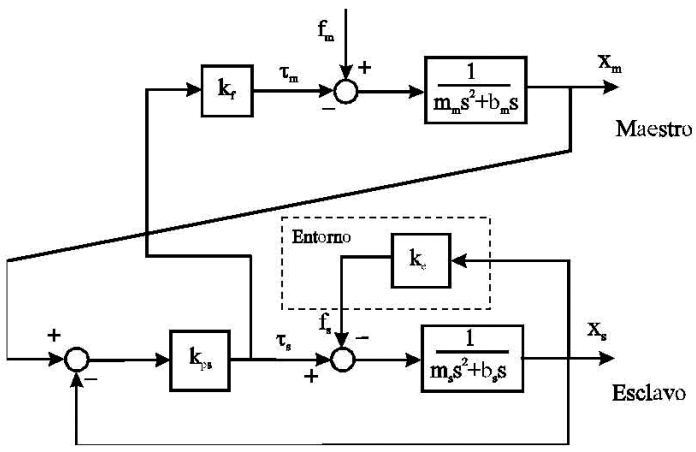

(f) Diagrama de control fuerza - fuerza

Figura 6. Diagramas de control de dispositivos bilaterales de una articulación [34].

dad virtual, como visualizador predictivo [37], simulando el ambiente de trabajo del robot para visualizar los movimientos que el esclavo realizará; En este caso se requiere tener amplio conocimiento del entorno remoto, de la dinámica del dispositivo esclavo, y además es 
necesario que las variables desconocidas del ambiente remoto cambien muy lentamente; de esta manera aunque se elimina absolutamente el problema del retardo de la señal, el correcto funcionamiento se obtiene solo para espacios de tarea sencillos y de poca precisión.

Cuando además de la realimentación de información visual, se dispone de realimentación de fuerza con grandes retardos; una metodología utilizada es enviar esta señal a la mano que no esta ocupada con el dispositivo maestro, ya que si la señal esta muy retrasada y se realimenta sobre miembro que opera el dispositivo maestro, el operario va a reaccionar a una fuerza, que muy posiblemente no este interactuando actualmente con el dispositivo esclavo [34].

Para minimizar la cantidad de datos enviados por el canal de información, se debe dar cierto nivel de autonomía al dispositivo remoto [42], de tal forma que el operador en lugar de indicar una trayectoria al robot, le indica una tarea completa, en este sentido, el operario pasa a ser un observador de la tarea, y en caso de error es él, el responsable de detenerla. Niemeyer [43] propone en su tesis doctoral la utilización de variables de onda, para modelar el sistema dinámico del medio de comunicación, aunque para un buen funcionamiento de este modelo se requieren características especiales de la respuesta temporal de la planta. Las variables tipo onda codifican la información e inmediatamente incluyen robustez a los retardos de señal. En su tesis realiza un trade-off entre la transparencia de la reflexión de fuerza y la velocidad de funcionamiento del sistema total.

\section{APLICACIONES}

Aplicaciones para manipuladores teleoperados existen bastantes, sin embargo vamos a citar unas cuantas:

Salcudean [44] desarrolla un robot para realizar tele-ecografía, este robot cuenta en su dispositivo esclavo con 6 grados de libertad y tiene reflexión de fuerza, es utilizado para distancias cortas con retardos de señal muy pequeños. Utiliza un método de control óptimo llamando $\mathrm{H}-\infty$.

Dentro del campo de la tele-ecografía, Randal [45] propone un robot para realizar ecografías 3D, con tres grados de libertad, en la actualidad, el sistema es utilizado en la realización de ecografías de la vena carótida, pero se piensa extender su uso a otros lugares del cuerpo. Stoll [46] también propone un robot para realización de teleecografía con tres grados de libertad. Las imágenes son mostradas al operario en tiempo real y este busca su objetivo a partir de estas. Este robot no posee reflexión de fuerza.

Cabe la pena mencionar el trabajo de Cavosoglu [47] que desarrolla un dispositivo para realizar laparoscopias. Otra aplicación existente fue desarrollada por Yang [48] quien realiza un manipulador con fines educativos, para usuarios que no pueden acceder a un manipulador real.

\section{CONCLUSIONES}

En el presente artículo se pueden apreciar los distintos métodos de control aplicados actualmente a los manipuladores de varios grados de libertad. El control basado en técnicas conven- 
cionales tiene ventajas como la velocidad de respuesta, una matemática bien conocida y además es liviano computacionálmente, no obstante estos controladores son incapaces de adaptarse a cambios de la planta.

La lógica difusa es un método basado en el conocimiento de un experto, puede manejar tanto sistemas lineales como no lineales, parámetros desconocidos y puede combinarse con otras técnicas, como redes neuronales y algoritmos genéticos, esta técnica permite adaptación del controlador a cambios en la planta.

Los controladores de manipuladores basados en redes neuronales que han sido probados son relativamente nuevos, debido a la falta de recursos computacionales suficientemente rápidos para implementar este tipo de control en tiempo real; por lo tanto es un área de investigación de rápido crecimiento.

Con el fin que un operario pueda trabajar con mayor destreza en ambientes teleoperados, se utilizan dispositivos bilaterales, estos aparatos permiten que el operador perciba las fuerzas que actúan en el entorno remoto.

Se puede decir, que un sistema de teleoperación ideal debe tener una realimentación de fuerza que garantice la estabilidad del dispositivo remoto para cualquier entorno, al tiempo que proporciona al usuario una inmersión total en el trabajo desarrollado.

En el caso de los sistemas teleoperados, las técnicas difieren dependiendo de la longitud del retardo, para retrasos pequeños no se requiere que el sistema manipulado tenga tanta autonomía como uno con retardos grandes, inclusive cuando el retardo es muy grande se suele simular el comportamiento del sistema en un entorno virtual, y dejar el control en tiempo real del lado del dispositivo remoto.

\section{REFERENCIAS}

[1] J. J. Craig, Introduction to Robotics: Mechanics and Control, P. Hall, Ed., 2003.

[2] A. Barrientos, L. F. Peñín, C. Balaguer, and R. Aracil, Fundamentos de Robótica. McGrawHill, 1998.

[3] Y.-C. Hsu, G. Chen, and H.-X. Li, "A fuzzy adaptive variable structure controller with application to robot manipulators," IEEE Transactions on systems, man and cybernetics, vol. 31, no. 3, June 2001.

[4] J. S. Albus, "A new approach to manipulator control: The cerebellar model articulation controller," J. of Dynamic Sys., Measurement and Control, ASME, pp. 220-227, 1975.

[5] J.-H. rya, D.-S. Kwon, and B. Hannaford, "Control of a flexible manipulator with noncollocatet feedback: time-domain passivity approach," IEEE Transactions ont Robotics, vol. 20, no. 4, pp. 776 - 780, August 2004.

[6] D. Moschini and P. Fiorini, "Performance of robotic teleoperation system with flexible slave device." " Proceedings of International Conference on Robotics and Automation (ICRA) (2004), 2004.

[7] A. Albu-Schaffer, F. Christian Ott, and G. 
Hirzinger, "Cartesian impedance control of redundant robots: Recent results with the dlr-lightweight-arms," ICRA, pp. 3704-3709, 2003.

[8] K.-S. Chang and O. Khatib, "Manipulator control at kinematic singularities: A dynamically consistent stategy," Proc. IEEE/RSJ Int. Conference on Intelligent Robots and Systems, vol. 3, pp. 84-88, 1995.

[9] A. A. M. Rodney G. Roberts, "Nearest optimal repeatable control strategies for kinematically redundant manipulators," IEEE Transactions on robotics and automation, vol. 8, no. 3, pp. 327-337, June 1992.

[10] S. 'Tong, H.-X. Li, and G. Chen, "Adaptive fuzzy decentralized control fo a class of largescale nonlinear systems," IEEE Transactions on systems, man and cybertnetics, vol. 34, pp. 770775, 2004.

[11] S. Tong, H.X.Li, and G. Chen, "Adaptive fuzzy decentralized control for a class of largescale nonlinear systems," IEEE Trans. on Sys. Man Cybern., vol. PP, p. 1096, 2003.

[12] J. W.Chang, Y.H.Joo, and GC, "Output feedback fuzzy control for uncertain nonlinear systems," ASME Journal of Dynamic Systems, Measurement and Control, vol. 125, pp. 521530, 2003.

[13] C. Ham, Z. Qu, and R. Johnson, "Nonlinear robust fuzzy control for robot manipulators," IEE: Control Theory and Applications, vol. 147, 2000.

[14] G. R.Ranganathan, H.A.Malki, "Fuzzy pre- dictive pi control for processes with large time delay," Expert Systems, vol. 19, pp. 21- 23, 2002.

[15] J.Carvajal, GC, and H.Ogmen, "Fuzzy pid controller: Design, performance evaluation, and stability analysis," J. of Inform. Sci., vol. 123, pp. 249-270, 2000.

[16] J.Lu, GC, and H.Ying, "Predictive fuzzy pid control: Theory, design, and simulation," Information Science, vol. 137, pp. 157-187, 2001.

[17] W.M.Tang, GC, and R.D.Lu, "A modified fuzzy pi controller for a flexible-joint robot arm with uncertainties," J. of Fuzzy Sets and Systems, vol. 118, pp. 109-119, 2001.

[18] H.X.Li and GC, "Dual features of conventional fuzzy logic control," Acta Automatica Sinica, vol. 27, pp. 1-13, 2001.

[19] D.Misir, H.Malki, and GC, "Design and analysis of a fuzzy proportional-integralderivative controller," J. of Fuzzy Sets and Systems, vol. 79, pp. 297-314, 1996.

[20] H.Malki, D.Misir, D.Feigenspan, and GC, "Fuzzy pid control of a flexible-joint robot arm with uncertainties from time-varying loads," IEEE Trans. on Contr. Sys. Tech., vol. 5, pp. 371-378, 1997.

[21] M. T. Eskil, M. O. Efe, and O. Kaynak, "'Tnorm adaptation in fuzzy logic systems using genetic algorithms," " 1999.

[22] K.S.Tang, K.F.Man, GC, and S.Kwong, "An optimal fuzzy pid controller," IEEE Trans. on Indus. Elect., vol. 48, pp. 757-465, 2001. 
[23] Y. Zhang, J. Wang, and Y. Xia, "A dual neural network for redundancy resolutin of kinematically redundant manipulators subject to joint limits and joint velocity limits," IEEE transactions on neural netwoks, vol. 14, no. 3, pp. 658-667, 2003.

[24] S. Hu, M. H. A. Jr., and H. Krishnan, "Online neural network compensator for constrained robot manipulators." " Asian Control Conference? 2000, 4-7 July 2000, Shanghai, China., 2000.

[25] C.Wang, GC, and S.S.Ge, "Smart neural control of uncertain nonlinear systems,"Int. J. of Adaptive Control and Signal Processing, vol. 17, pp. 467-488, 2003.

[26] Q. Gan and E. Rosales, "Cmac with linear functional weights," in In Proc of the 13th IFAC Symposium on Systems and Identification (SYSID'2003), pages 1838-1843, The Netherlands, August 2003., 2003.

[27] P. E. Almeida and M. G. Simoes, "Parametric cmac networks: Fundamentals and applications of a fast convergence neural structure," IEEE Transactions on industry applications, vol. 39, no. 5, pp. 1551-1557, 2003.

[28] S. Sayil, "A combine algorithm for a cmac network," PAU Journal of Engineering Science, 2001.

[29] C. M. Clark and J. K. Mills, "Robotic system sensitivity to neural network learning rate: Theory, simulation and experiments," The International Journal of Robotics Research, vol. 19, no. 10, pp. 955-968, 2000.
[30] X.F.Liao, GC, and E.N.Sanchez, "Lmibased approach for asymptotically stability analysis of delayed neural networks," IEEE Trans. on Circ. Sys.-I, vol. 19, pp. 1033- 1039, 2002.

[31] A. Bogdanov and A. Timofeev, "Robust optimal neural control of robots," Proceedings of IEEE international conference IJCNN99, , IEEE, Washington, D.C., Jul, 1999, pdf., December 1999.

[32] P. van der Smagt, "Cerebellar control of robot arms," Connections Science, vol. 10, no. 10, pp. 301-320, Dicember 1998.

[33] M. T. Rosenstein, "Learning to expoit dynamics for robot motor coordination," Ph.D. dissertation, University of Massachusetts, 2003.

[34] J. M. A. Poveda, "Control bilarteral por convergencia de estados de sistemas teleoperados con retardos en las transmisión," Ph.D. dissertation, Universidad Miguel Hernández, 2003.

[35] J. Vertut and P. Coiffet, Teleoperation and Robotics. Evolution and Development. Kogan Page, 1985.

[36] W. R. Ferrell, "Delayed force feedback," IEEE Transactions on Human FActors in Electronics, vol. 8, pp. 449-455, 1966.

[37] P. Batsomboon, S. Tosunoglu, and D. W. Repperger, "A survey of telesensation and teleoperation technology with virtual reality and force reflection capabilities," International Journal of Modeling and Simulation, vol. 20, pp. 79-88, 2000. [Online]. Available: 
[38] J. Yan and S. Salcudean, "Teleoperation controller design using h-optimization with application to motion -scaling," IEEE Transaction on Control Systems technology, vol. 20, 1996.

[39] G. Feng and G. Chen, "Adaptive control of discrete-time chaotic systems: a fuzzy control approach," Chaos Solitns \& Fractals, vol. 23, pp. 459-467, 2005.

[40] R. Aracil, J. Bario, M. Ferre, and J. M. Bogado, "Identificación y modelado de un sistema maestro-esclavo para teleoperación," in XXV Jornadas de Automática, 2004.

[41] R. A. C. Bianchi, B. S. M. Jr, F. C. Ferrez, and A. H. R. C. Rillo, "Operation of a robotic manipulator through the www," FEI, 1998.

[42] M. R. Stein, "Behavior -based control for time-delayed teleoperation," Ph.D. dissertation, University of Pennsylvania, 1994.

[43] G.Ñiemeyer, "Using wave variables in time delayed froce reflecting teleoperation," Ph.D. dissertation, Massachusetts Institute of Technology, 1996.

[44] S. Salcudean, W. Zhu, P. Abolmaesumi, S. Bachmann, and P. Lawrence, "A robot system for medical ultrasound," 1999.

[45] R. P. Goldberg, M. Dumitru, R. H. Tailor, and D. Stoinanovici, "A modular robotic system for ultraound image acquisition," Proceeding of the 4th INternational Conference on Medical Image Computing and Computer-Assisted Intervention, vol. 2208, pp. 1430-1432, 2001.
[46] J. Stoll, P. Dupont, and R. Howe, "Ultrasound-based servoing of manipulators for telesurgery," Telemanipulator and Telepresence Technologies VIII Conference. Proceeding of the SPIE, vol. 4570, 2001.

[47] F. T. S. S. S. M. Cenk Cavosoglu, Wintrhop Williams, "Robotics for telesurgery: Second generation berkeley / ucsf laparoscopic telesurgical worksatation and looking rowards the future applications," in In Proceeding of the 39th Allerton Conference on Communication, Control and Computing, 2001.

[48] X. Yang, Q. Chen, D. C. Petriu, and E. M. Petriu, "Internet-based teleoperation of a robot manipulator for education," Haptic, Audio and Visual Environments and Their Applications, 2004. HAVE 2004. Proceedings. The 3rd IEEE International Workshop on, pp. 7 - 11, 2004. 